\title{
Purpose and procedure
}

The purpose of Evidence-Based Mental Health is to alert clinicians working in the field of mental health to important and clinically relevant advances in treatment (including specific interventions and systems of care), diagnosis, aetiology, prognosis/outcome research, quality improvement, continuing education, economic evaluation, differential diagnosis, and qualitative research. We will do this by selecting original and review articles whose results are most likely to be accurate and clinically useful. The articles are then summarised in value added abstracts and a commentary by a clinical expert is added.

Our target audience is psychiatrists, psychologists, nurses, occupational therapists, pharmacists, and other professionals whose clinical work can be enhanced by up to date knowledge of research in mental health. The nature of work in mental health is multidisciplinary and the aim of Evidence-Based Mental Health will be to inform mental health clinicians from all disciplines of highly relevant developments within the overall field. Evidence-Based Mental Health will cover articles concerned with a broad range of mental health problems including adults, children, older adults, people with learning disabilities, people with head injuries, drug and alcohol problems, personality disorders, and individuals who have developed psychiatric and psychological problems as a result of trauma, and psychological or psychiatric problems of people with physical health problems.

The procedures that are used to select and abstract journal articles are based closely on those developed by our sister journal Evidence-Based Medicine. These procedures are:

- Selecting, using prestated, empirically derived criteria, the best original and review articles on the causes, course, diagnosis, prevention, treatment, quality of care, economics, or qualitative research of disorders in mental health

- Introducing these articles with declarative titles stating the clinical bottom line and summarising them in structured abstracts that describe their questions, methods, and results

- Adding brief commentaries prepared by clinical experts to place each study in its clinical healthcare context

- Disseminating these summaries to clinicians as soon as possible after the publication of the original article.

Evidence-Based Mental Health is published quarterly by the BMJ Publishing Group under the editorship of Drs John Geddes at the University of Oxford, Shirley Reynolds at the University of East Anglia, David Streiner at the University of Toronto, and Peter Szatmari, and $\mathrm{R}$ Brian Haynes (coordinating editor) at McMaster University in Canada. The Health Information Research Unit of the Department of Clinical Epidemiology and Biostatistics at McMaster University hosts the editorial office for the service.

The following journals are regularly reviewed:

Acta Psychiatrica Scandinavica

Addiction

Age and Ageing

American Journal of Psychiatry

American Psychologist

Archives of General Psychiatry

Australian and New Zealand Journal of Psychiatry

BMJ

Behaviour Research and Therapy

Behaviour Therapy

British Journal of Clinical Psychology

British Journal of Psychiatry

Canadian Journal of Psychiatry

Child Development

Clinical Psychology Review

Cochrane Library
Cognitive and Behavioral Practice

Developmental Medicine and Child Neurology

General Hospital Psychiatry

Health Psychology

International Journal of Behavioural Medicine

International Journal of Geriatric Psychiatry

JAMA

Journal of Abnormal Child Psychology

Journal of Abnormal Psychology

Journal of Affective Disorders

Journal of Autism and Developmental Disorders

Journal of the American Academy of Child and Adolescent Psychiatry

Journal of the American Geriatrics Society

Journal of Child and Adolescent Psychopharmacology

Journal of Child Psychology and Psychiatry and Allied Disciplines

Journal of Clinical and Experimental Neuropsychology

Journal of Clinical Psychiatry

Journal of Clinical Psychopharmacology

Journal of Consulting and Clinical Psychology

Journal of Neurology, Neurosurgery, and Psychiatry

Journal of Neuropsychiatry and Clinical Neurosciences

Journal of Psychosomatic Research

Lancet

New England Journal of Medicine

Psychiatric Services

Psychiatry Interpersonal and Biological Processes

Psychological Bulletin

Psychological Medicine

Psychology and Aging

Psychosomatic Medicine

Schizophrenia Bulletin

Social Science and Medicine

This list of journals is subject to modification based on the relative performance of each journal according to the criteria set out below; we also assess journals nominated by our readers.

\section{Criteria for selection of articles for abstracting}

All articles in a journal issue are considered for abstracting if they meet the following criteria:

\section{BASIC CRITERIA}

- Original or review articles in English and about humans

- About topics that are important to the practice of clinicians in the broad field of mental health.

\section{Studies of prevention or treatment must meet these additional} criteria:

- Random allocation of participants to comparison groups

- Follow up (end point assessment) of at least $80 \%$ of those entering the investigation

- Outcome measure of known or probable clinical importance

- Analysis consistent with study design.

Studies of diagnosis must meet these additional criteria:

- Inclusion of a spectrum of participants, some, but not all of whom have the disorder or derangement of interest

- Diagnostic (gold) standard (eg, diagnosis according to DSM-IV or ICD-10 criteria after assessment by clinically qualified interviewer) preferably with documentation of reproducible criteria for subjectively interpreted diagnostic standard (eg, report of statistically significant measure of agreement among observers)

- Each participant must receive both the new test and some form of the diagnostic standard

- Interpretation of diagnostic standard without knowledge of test result 
- Interpretation of test without knowledge of diagnostic standard result

- Analysis consistent with study design.

\section{Studies of prognosis must meet these additional criteria:}

- Inception cohort (first onset or assembled at a uniform point in the development of the disease) of individuals, all initially free of the outcome of interest

- Follow up of at least $80 \%$ of patients until the occurrence of a major study end point or to the end of the study

- Analysis consistent with study design.

\section{Studies of causation must meet these additional criteria:}

- Observations concerning the relationship between modifiable exposures and putative clinical outcomes

- Prospective data collection with clearly identified comparison group(s) for those at risk of the outcome of interest (in descending order of preference, from randomised controlled trials, quasi-randomised controlled trials, non-randomised controlled trials, cohort studies with case by case matching or statistical adjustment to create comparable groups, or nested case control studies)

- Masking of observers of outcome to exposure (this criterion is assumed to be met if the outcome is objective)

- Analysis consistent with study design.

\section{Studies of quality improvement and continuing education} must meet these additional criteria:

- Random allocation of participants or units to comparison groups

- Follow up of at least $80 \%$ of participants

- Outcome measure of known or probable clinical importance

- Analysis consistent with study design.

Studies of the economics of healthcare programmes or

interventions must meet these additional criteria:

- The economic question addressed must be based on comparison of alternative diagnostic or therapeutic services or quality improvement strategies

- Activities must be compared on the basis of the outcomes produced (effectiveness) and resources consumed (costs)

- Evidence of effectiveness must be from a study (or studies) of real (not hypothetical) patients, which meets the journal criteria for diagnosis, treatment, quality improvement, or a review article

- Results should be presented in terms of the incremental or additional costs and outcomes of one intervention over another

- Where there is uncertainty in the estimates or imprecision in the measurement, a sensitivity analysis should be done.

\section{Studies of differential diagnosis must meet these additional criteria:}

- Cohort of patients who present with a similar, initially undiagnosed but reproducibly defined clinical problem

- Clinical setting, including referral filter, is explicitly described

- At least $80 \%$ of initially undiagnosed patients are followed up for $\geqslant 1$ month for acute disorders or $\geqslant 1$ year for chronic or relapsing disorders.

Review articles must meet these additional criteria:

- Statement of the clinical topic

- An identifiable description of the methods indicating the sources

- Explicit statement of the inclusion and exclusion criteria applied for selecting articles for detailed review
- At least one article included in the review must meet the above noted criteria for treatment, diagnosis, prognosis, causation, quality improvement, or the economics of healthcare programmes.

Qualitative studies must meet these additional criteria:

- Content must relate to how people feel or experience certain situations, specifically those situations that relate to health care

- Data collection methods must be appropriate for qualitative studies (eg, unstructured interviews, semi-structured interviews, participant observation of people in natural settings, focus groups, review of documents or text)

- Data analyses must be appropriate for qualitative studies (ie, the primary analytical mode is inductive rather than deductive; units of analysis are ideas, thoughts, concepts, phrases, incidents, or stories which are ultimately classified into categories or themes).

These criteria are subject to modification if, for example, it is found feasible to apply higher standards that increase the validity and applicability of studies for clinical practice. The objective of Evidence-Based Mental Health is to abstract only the best literature, consistent with a reasonable number of articles making it through the filter.

Evidence-Based Mental Health has 3 related journals: EvidenceBased Medicine, ACP Journal Club, and Evidence-Based Nursing. Abstracts in their entirety, or a modification thereof, may appear in this journal and a footnote indicating this will appear with each piece. This arrangement increases the number of journals that are regularly searched.

Articles meeting the criteria set out above are abstracted according to the procedure for more informative abstracts, ${ }^{1}$ with the following modification: abstracts can be up to 440 words in length; and each is reviewed by an expert in the content area covered by the article and a commentary is added to provide the context of the article in the research that has preceded it; to provide any important methodological problems that affect interpretation; and to provide recommendations for clinical application. The author of the article is given an opportunity to review the abstract and commentary before publication. If there is not enough space to accommodate abstracts for all articles that meet review criteria, citations for the extra articles will be noted in the section of the journal titled Other Articles Noted.

\section{Developing additional criteria for reviewing and abstracting articles for Evidence-Based Mental Health}

Most mental health research studies can be judged using the criteria developed by Evidence-Based Medicine. However, there are other high quality studies answering different kinds of clinical questions for which there are currently no criteria. Therefore, an important and urgent issue for Evidence-Based Mental Health to resolve is how to establish criteria to judge the scientific validity of a broader range of empirical research. An important recent response to this issue is criteria for evaluating qualitative studies. Other investigations we wish to develop criteria for are prevalence studies, and studies of process in the treatment of mental health problems. We think that the development of these criteria will be achieved most effectively by a collaborative process through debate and discussion within Evidence-Based Mental Health. We invite readers to participate in this process by corresponding with the editors.

1 Haynes RB, Mulrow CD, Huth EJ, et al. More informative abstracts revisited. Ann Intern Med 1990;113:69-76. 